\section{European Network Supports Interaction Among Materials Research Labs}

In order to encourage stronger interaction among the most active laboratories involved in materials research, the European Community Commission (ECC) has initiated a special "European Networks" fund. Financial support will be provided to groups of laboratories within the European community to facilitate and encourage the coordination of their research efforts. The first year of this activity will be devoted to meetings and colloquia which have two objectives: (1) to compose for each specific subject a document which focuses on the state-of-the-art in that particular field, and (2) to elaborate a research proposal which would support a common effort. A substantial financial effort foreseen for the second year would launch a number of research projects chosen from among the proposals which are elaborated in the framework of the European Network.

Nine groups have received financial support through E-MRS, forming the European Network on Advanced Materials. The structure and the goals of these nine groups are described briefly here.

\section{Laser Chemistry}

The coordinators of this group are K.L. Kompa, MPI Quantenoptic, Garching; and E.F. Krimmel, Forschungslaboratorien, Siemens, Munchen, West Germany.

This group will focus on techniques which use light for materials processing. The name "laser chemistry" was chosen because many processes have been realized only since lasers as intensive light sources have become available. In this new method, reactions among atoms, molecules, and/or radicals are induced by light, if its intensity exceeds a certain critical value. Laser chemistry permits, for instance, the production of new materials containing components which do not intermix when applying conventional diffusion and high temperatures.

In spite of many important and interesting results achieved with the new technique, it is felt that more knowledge is needed to understand the fundamental nature and kinetics of individual processes involved in order to promote its industrial development and the production of new materials and novel devices.

More than 50 institutions in Europe are active in laser chemistry. Since laser chemistry is still in its initial stages of development, an intensive definition phase is required in order to establish an efficient network of intercommunicating institutions and a well-selected research and development program.

The topics of laser chemical processes to be discussed include the structured depo- sition of thin films or multilayers of semiconductors, metals or new ceramics on substrates for microelectronic devices, structured etching of such devices, catalysts or processing of polymers or biological materials, and the analysis of processed materials.

\section{Solid-State Batteries}

The coordinator of this group is $M$. Balkanski, Laboratoire de Physique des Solides, Université Pierre et Marie Curie, Paris, France.

This group will develop research on materials relevant to solid-state microbattery structures. Attention will be focused on materials such as fast ion conducting solid electrolytes and borate glasses, and layered compound intercalation materials. The research program involves fundamental studies (theoretical and experimental), materials preparation and characterization, and building and testing of batteries. Laboratories from five European countries are involved in this program.

\section{Crystal Growth and \\ Solidification of Metals}

The coordinator of this group is $H$. Fredriksson, Department of Casting of Metals, Royal Institute of Technology, Stockholm, Sweden.

This network will be a forum of scientists to discuss and exchange the latest results in casting, crystal growth, and solidification. It will also be a forum where new international projects are created and studied. During the last year computer simulation of casting and solidification processes was of great interest to the industry, so it has been deemed necessary to develop different types of computer programs. Cooperation among laboratories will make it possible to exchange programs and ideas.

Another primary goal of the network will be to organize symposia and summer schools to spread the program to a wider range of research and industrial groups. Nine laboratories from six different countries are participating in this network.

\section{New Methods in Metastable \\ Alloy Production}

The coordinating committee of this network was formed by J. Bottiger, Arthus, Denmark; B. Stritzker, Jülich, West Germany; and M. von Allmen, Bern, Switzerland.

The aim is to organize a concerted European effort toward the technological development and physical understanding of innovative methods to produce metastable materials, including supersaturated solutions, micro- and nanocrystalline alloys, and glassy metals. Many of the unique general characteristics of such materials are known, but the techniques to produce them are still largely experimental. Techniques of interest are ion beam irradiation, laser beam irradiation, solid-state reactions, cold vapor deposition, melt spinning, and powder methods in addition to solution-chemical processes. Nine laboratories from six different countries are involved in this project.

\section{High Resolution and High Sensitivity Analyses of Semiconductors}

The coordinators of this network are A.G. Cullis, Royal Signals and Radar Establishment, Great Malvern, United Kingdom; and E. Sirtl, Heliotronic GmbH, Burghausen, West Germany.

High resolution and high sensitivity analyses of semiconductors are fundamental to semiconductor investigations, from basic research to device development. In a first step, this project will concentrate on the introduction, behavior, and effects of $\mathrm{H}$ in crystalline silicon. Different analytical techniques will be used. In particular, high resolution transmission electron microscopy will be applied to imaging lattice defects in H-doped semiconductors. Several high sensitivity techniques will also be applied: bulk analysis by effusion, SIMS, or neutron activation; structural analysis by $x$-ray, neutron, or $\gamma$-diffraction, and by channeling techniques like RBS; electronic analysis (deep level spectroscopy, carrier lifetime measurements); and electronic analysis of interfaces (e.g., $\mathrm{Si}_{-}-\mathrm{SiO}_{2}$ interface or grain boundaries).

The research program will concentrate on the following: (1) thermodynamic properties of $\mathrm{H}$ in semiconductors, (2) interaction of $\mathrm{H}$ with lattice and impurities, (3) interaction of $\mathrm{H}$ with point defects, and (4) interaction of $\mathrm{H}$ with line defects and interfaces or grain boundaries, respectively. A large number of laboratories from all European countries are candidates for participation in this network.

\section{Ion Beam Processing of \\ Materials for Electronics}

The coordinator of this network is G.G. Bentini, CNR, Istituto Lamel, Bologna, Italy.

The network on ion beam processing in materials for electronics involves the participation of several European laboratories which, in the last several years, have been among the most active centers in ion implantation on semiconductors. Included is a project to develop at least one high energy/high current European implantation facility where the necessary sample irradiation can be performed. Also recommended is support of the development of prototypes of the European high energy implanters. The goal of this network is not only to develop cooperative research on advanced processes, but also to extend this to the realization and use, on a European scale, of high technology equipment having both scientific and commercial value.

Continued 
Sixty scientists in nine institutes from five countries are available for this program.

\section{II-VI Telluride-Based Semiconductors}

The coordinator of this network program is $\mathrm{R}$. Triboulet, Laboratoire de Physique des Solides, CNRS, Meudon, France.

The II-VI telluride compounds and alloys are being investigated for application to the detection of radiation, nuclear particles for $\mathrm{CdTe}$, and infrared radiation for the mercury cadmium ternary alloys. The research program is based on investigations aimed at developing a method for thin film growth by decomposition of organometallics. The objectives of this program also encompass the physics of doping, including the introduction of impurities ( $\mathrm{Al}$, In) by ion implantation, study of the impurity compensation and of the electronic levels associated with the impurities according to their incorporation into the lattice, and studies on the alloys. Laboratories from five countries will take part in this project.

\section{Biomaterials}

The coordinator of this network is D. Muster, CHU, Strasbourg, France.

Biomaterials are now used for rehabilitation, convenience, amenity, and aesthetics. The problems they raise involve aspects of the life sciences, engineering, and the socioeconomic sciences. While the main aim of this multidisciplinary applied research is to improve the reconstruction of organs and tissues, it will also target the effects of medicines more accurately and help enhance basic knowledge of the interaction between living structures and chemical compounds.

This joint effort will be based on three aims - training, research, and development. The following subjects seem most likely to complement one another and generate new ideas: development of new materials specifically designated for biological use, optimization of traditional materials and study of their biocompatibility, and monitoring of implants under biological stress. The partners in this project are laboratories and institutions from six European countries.

\section{Gallium Arsenide}

The coordinator of this network is H.S. Rupprecht, LAF, Freiburg, West Germany.

Due to its specific advantages, GaAs has allowed the development of various novel electronic and optoelectronic devices. Materials research is needed to improve and stabilize the present achievements. The proposed program will have three directions:
(1) semi-insulating bulk GaAs: crystal growth, stoichiometry control, wafer manufacturing and handling, identification of point and extended defects, impact of defects and device processes on materials and device properties, device related routine wafer checks, chemical trace analysis, and the establishment of specifications;

(2) epitaxial layers: reliable deposition techniques such as MBE, MOCVD, and MOMBE; low pressure MOCVD for production, ultrapure metalorganic and doping gases; and topographic characterization techniques; and

(3) contact metallurgy: surface and interface phenomena, and phase equilibria. Sixteen institutions from nine European countries are involved in this program.

The European Network on Advanced Materials is now funded for an initial period of one year. At the end of this year a report describing the state-of-the-art in the nine areas enumerated here and describing specific research and development projects will be presented to the ECC. The projects selected and financed by the technical commission will then enter the active phase of research. This action is expected to bring a vital momentum to European materials research.

M. BALKANSKI

\section{E-MRS PROCEEDINGS}

R-1

Poly-Micro-Crystalline and Amorphous Semiconductors Editors: P. Pinard, S. Kalbitzer, 1984

MRS Members $\$ \$ 52.00 \quad$ List $-\$ 62.00$

R-2 Amorphous Metals and Non-Equilibrium Processing Editor: $M$. von Allmen, 1984

MRS Members $-\$ 40.00 \quad$ List $-\$ 50.00$

R-3 Induced Defects in Insulators

Editor: P. Mazzoldi, 1984

MRS Members $-\$ 34.00 \quad$ List $-\$ 44.00$

R-4 Energy Beam Solid Interactions and Transient Thermal Processing

Editors: V.T. Nguyen, A.G. Cullis, 1985

MRS Members $\$ 70.00 \quad$ List $-\$ 80.00$

R-5 Materials under Extreme Conditions

Editors: H. Ahlborn, H. Fredriksson, E. Luscher, 1985

MRS Members - $\$ 35.00 \quad$ List $-\$ 45.00$

R-6 Semiconductor Quantum Well Structures and

Superlattices

Editors: K. Ploog, N.T. Linh, 1985

MRS Members $-\$ 42.00 \quad$ List $-\$ 52.00$

R-7 Advanced Materials Research and Developments for Transport: Light Metals

Editors: R.J.H. Wanhill, W.J.G. Bunk, J.G. Wurm, 1985

MRS Members $\$ \$ 52.00 \quad$ List $-\$ 62.00$
R-8 Advanced Materials Research and Developments for Transport: Composites

Editors: P. Lamicq. W.J.G. Bunk, J.G. Wurm, 1985

MRS Members $-\$ 52.00 \quad$ List $-\$ 62.00$

R-9 Advanced Materials Research and Developments for Transport: Ceramic Coatings for Heat Engines,

Editors: I. Kvernes, W.J.G. Bunk, J.G. Wurm, 1985

MRS Members - $\$ 52.00 \quad$ List $-\$ 62.00$

R-10 Magnetic Thin Films

Editor: R. Krishnan, 1986

MRS Members $-\$ 31.00 \quad$ List $-\$ 41.00$

R-11 Laser Processing and Diagnostics (II)

Editors: D. Bauerle, K.L. Kompa, L. Laude, 1986

MRS Members $-\$ 49.00 \quad$ List $-\$ 59.00$

R-12 Dielectric Layers in Semi Conductors: Novel

Technologies and Devices,

Editor: G. Bentini, 1986

MRS Members $-\$ 56.00 \quad$ List $-\$ 66.00$

R-13 Advanced Materials for Telecommunications

Editors: P.A. Glasow, Y.I. Nissim, J.P. Noblanc, J. Speight, 1986

MRS Members - $\$ 75.00 \quad$ List $-\$ 85.00$

R-14 State of the Art of Computer Simulation of Casting and Solidification Processes,

Editor: H. Fredriksson, 1986

MRS Members $-\$ 52.00 \quad$ List $-\$ 62.00$ 